\title{
Analysis of High Birefringence of Four Types of Photonic Crystal Fiber by Combining Circular and Elliptical Air Holes in Fiber Cladding
}

\author{
Yuan-Fong Chau, ${ }^{1}$ Han-Hsuan Yeh, ${ }^{1}$ and Din Ping Tsai ${ }^{2}$ \\ ${ }^{1}$ Department of Electronic Engineering, Ching Yun University, Jung-Li 320, Taiwan \\ ${ }^{2}$ Department of Physics and Center of Nanostorage Research, National Taiwan University, Taipei 10617, Taiwan \\ Correspondence should be addressed to Yuan-Fong Chau,yfc01@cyu.edu.tw
}

Received 1 October 2008; Accepted 8 December 2008

Recommended by N. Broderick

This paper presents a numerical study of high birefringence induced by four types (Type 1-4) of different sizes of elliptical air holes in photonic crystal fibers (PCFs). The numerical simulation is carried out by using the finite element method. The statistical correlations between the birefringence and the various parameters are obtained. Based on our results, the birefringence is found to be largely dependent on the variation of the normalized frequency, size ratio, effective area of the circular and elliptical air holes, and the ring number of cladding. Two of our suggested structures, Type 1 and Type 3, can considerably enhance the birefringence in PCFs leading to values as high as $7.697 \times 10^{-3}$ and $8.002 \times 10^{-3}$, respectively, which are much higher than that obtained by a conventional step-index fiber.

Copyright (c) 2008 Yuan-Fong Chau et al. This is an open access article distributed under the Creative Commons Attribution License, which permits unrestricted use, distribution, and reproduction in any medium, provided the original work is properly cited.

\section{INTRODUCTION}

Photonic crystal fibers (PCFs) guide the electromagnetic field by an arrangement of air holes that run down the entire fiber length. Recently, the most common cladding of PCFs has been investigated extensively [1-6], which consists of circular air holes, arranged in a triangular lattice with symmetric structure. For any fiber with rotational symmetry of order higher than two, a mode that has a preferred direction must be one of a pair of degenerate modes. Symmetry in PCFs implies the existence of doubly degenerate pairs of modes, that share the same propagation constant $(\beta)$ and free-space wavelength $(\lambda)$, so they must be degenerate. As a result, the observation of birefringence must be a result of asymmetry in the structure. These perturbations couple the modes that propagate at slightly different phase velocities, with the consequence that the polarization of light becomes unpredictable after a short propagation. To overcome this drawback, it is highly desirable to generate a large birefringence with a low scattering loss and at the same time to avoid significant perturbations in the cladding modes to cope with recent challenges and demands in fiber optic polarization control. When control over the polarization of light is crucial, high birefringence (up to about $5 \times 10^{-4}$ ) may be induced on purpose in conventional fibers by a number of techniques [7], and this reduces the coupling between the once degenerate modes. A number of recent studies indicate that PCFs with a preferred direction in their geometry could exhibit birefringence of about an order of magnitude higher than that obtained with conventional techniques [8-10].

As described in our previous work [11], the key point to induce high birefringence $B$ is to destroy the symmetry of the structure, and make the mode of the two orthogonal polarization states different. Usually, the anisotropy in PCFs can be introduced using elliptical air holes [12-16] and with asymmetric core [17] or asymmetric distribution of air holes in the cladding [18]. In order to combine asymmetry in both the core and the cladding, a PCF formed by a triangular lattice of elliptical air holes with double-defect fiber core is reported to yield a value of $B \sim 10^{-2}$ in [19]. Based on the structure asymmetry, the PCF structure in [20] is proposed 
by employing elliptical air holes in the fiber core (to induce high birefringence up to $10^{-2}$ order) but circular air holes in the fiber cladding (to reduce the confinement loss). However, the shape of the elliptical air holes needs a careful control in the fabrication process [20]. Ortigosa-Blanch et al. adopted an asymmetric cladding design [18] with the PCF consists of triangular lattice air holes, while one adjacent small central air hole is missing. Birefringence was introduced into the fiber only by a line defect of a small air hole array in the PCF center.

The structures formed by the elliptical air holes appear exhaustively complicated on the experience with fabrication of PCFs with varying hole sizes. Based on our previous works [11-13], high birefringence induced by complex (or binary) unit cell of circular and elliptical air holes PCFs is analyzed numerically in this paper using finite element method. Four types of PCFs are investigated. The origin of the birefringence is discussed in detail and its dependence on the structural parameters is analyzed. Furthermore, we discuss the influence of the rings of air holes and confinement loss on the influence of birefringence. Binary unit cell is the simplest way to form the complex cladding. The proposed structure has circular and elliptical air holes of two different sizes to replace the singular size of air holes in a PCF cladding, and is different from that proposed in conventional PCF designs. The birefringence of our structure is a result of the whole cladding asymmetry. The fiber core is a defect, which is formed by the omission of one circular air hole in the PCF center, which the mode field is well confined in the core region; thus, it is possible to create a high birefringence and low loss PCF. Together with the technological advancement in the fabrication of PCFs $[21,22]$, it is possible to fabricate our suggested PCF, but the technology for forming the complex cladding (different sizes of small and large air holes) may be challenging during fabrication of the proposed fiber.

\section{SIMULATION METHOD}

Among the full vectorial methods used in modeling PCFs [23-28], the finite-element method (FEM) is particularly effective for handling curved interfaces with high accuracy, and it is obviously a good choice for the analysis of combined circular and elliptical shape. The numerical method used in this study is FEM which is adequate for the analysis of general dielectric waveguide geometries. It has been already successfully applied to investigate dispersion properties of triangular and cobweb PCFs [29]. The fiber cross-section representation is very accurate as the domain is divided into subdomains with triangular or quadrilateral shape, where any refractive index profiles can be properly represented. Applying the variational FEM procedure to the curl-curl equation for magnetic field $\mathbf{H}$, one obtains an eigenvalue equation ( $[\mathbf{P}]-\beta^{2}[\mathbf{Q}]\{\mathbf{H}\}=0$, where $\{\mathbf{H}\}$ is the global magnetic field vector, $\beta$ is the propagation constant, and $[\mathbf{P}]$ and $[\mathbf{Q}]$ are both sparse matrices. The symmetry of the fiber cross-section is exploited to reduce CPU time and memory requirements. Solving the eigenvalue equation yields the effective indices of guided modes $n_{\text {eff }} \equiv \beta / k_{0}$, where $k_{0}=2 \pi / \lambda$ is the wave vector in free space. The modal birefringence of the fiber is then obtained by $B \equiv \mid n_{\mathrm{eff}}^{x}-$ $n_{\mathrm{eff}}^{y}$, where $n_{\mathrm{eff}}^{x}$ and $n_{\mathrm{eff}}^{y}$ are effective indices of the $x$ - and $y$-polarized fundamental modes, respectively. In order to model infinite PCF with two-dimensional-finite-geometry (i.e., to enclose the computational domain without affecting the numerical solution), it is necessary to use anisotropic perfectly matched layers (PMLs) which are placed in the contact with the outermost boundary.

\section{DESIGN AND RESULTS}

Four kinds of PCF cores, which are formed by the omission of different sizes of air hole in the PCF center, are analyzed for comparison, as shown in Figures 1(a)-1(d). For convenience, we name the corresponding fibers as Type 1 (omitting a small air hole in the center of the PCF, which has a small and a large elliptical air hole as a binary unit cell in the PCF cladding, see Figure 1(a)), Type 2 (the same as Type 1 , but omitting a large air hole in the center of the PCF, see Figure 1(b)), Type 3 (omitting a small air hole in the center of the PCF, which has a small elliptical and a large circular air holes as a binary unit cell in the PCF cladding, see Figure 1(c)), and Type 4 (omitting a large air hole in the center of the PCF, which has a small circular and a large elliptical air holes as a binary unit cell in the PCF cladding, see Figure $1(\mathrm{~d})$ ), respectively. In Types 1-4, each lattice point forms the cladding which consists of two different sizes of air holes with a pitch (center-to-center distance between the holes), $\Lambda=1.96 \mu \mathrm{m}$. As shown in Figures $1(\mathrm{a})$ and $1(\mathrm{~b})$ (Type 1 and Type 2 structures), $a_{1}$ and $b_{1}$ denote the half length of the large elliptical holes along $X$-(minor axis) and $Y$-(major axis) directions, respectively. In the same manner, $c_{1}$ and $d_{1}$ represent the ones with smaller elliptical ones. In Figure 1(c) (Type 3 structure), $c_{2}$ and $b_{2}$ denote the half length of the small elliptical holes along $X$ - and $Y$ directions, and $a_{2}$ represents the radius of large circular air hole. In Figure $1(\mathrm{~d})$ (Type 4 structure), $a_{3}$ and $b_{3}$ denote the half length of the large elliptical holes along $X$ - and $Y$ directions, and $c_{3}$ represents the radius of small circular air hole. We also define a coefficient $\eta=a_{1} / c_{1}=a_{2} / b_{2}=$ $a_{3} / c_{3}$ to determine the relationship between the large and small air-hole sizes. In addition, a solid silica jacket was added to increase the diameter and strength of this fiber. The refractive index of the background silica is set to be $n=1.45$.

We consider the birefringence of the fundamental mode and the modal birefringence $B$, which is defined above in Section 2. To illustrate the field profile of Type 1 and Type 2 PCFs, the fundamental mode with the parameters, $\Lambda=1.96 \mu \mathrm{m}, A / \Lambda^{2}=0.4, \eta\left(a_{1} / c_{1}\right)=5.5$, elliptical ratio $a_{1} / b_{1}=c_{1} / d_{1}=0.83$ at excitation wavelength $\lambda=1550 \mathrm{~nm}$, are shown in Figure 2, which shows the confinement of light in the PCFs. In Figure 2(a), the $X$ - and $Y$-polarized modes are strongly bound with the effective indices $n_{\text {eff }}^{x}=1.390869$ and $n_{\text {eff }}^{y}=1.383283$, respectively. This leads to a birefringence $B \equiv\left|n_{\text {eff }}^{x}-n_{\text {eff }}^{y}\right|=7.586 \times 10^{-3}$, which is much higher than the value of $3.7 \times 10^{-3}$ obtained from the previous PCF 


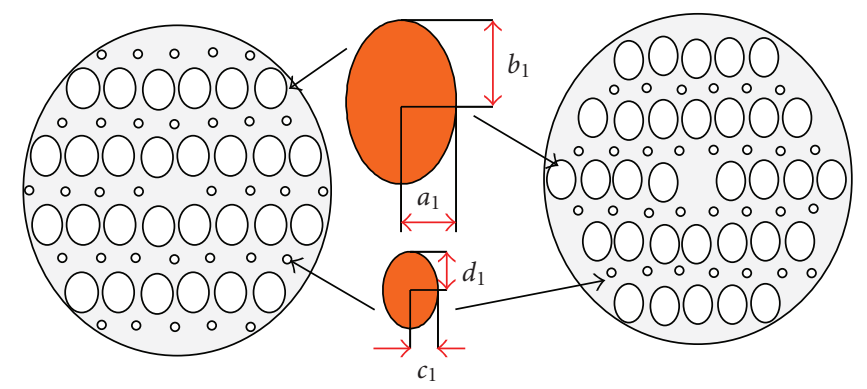

(a)

(b)

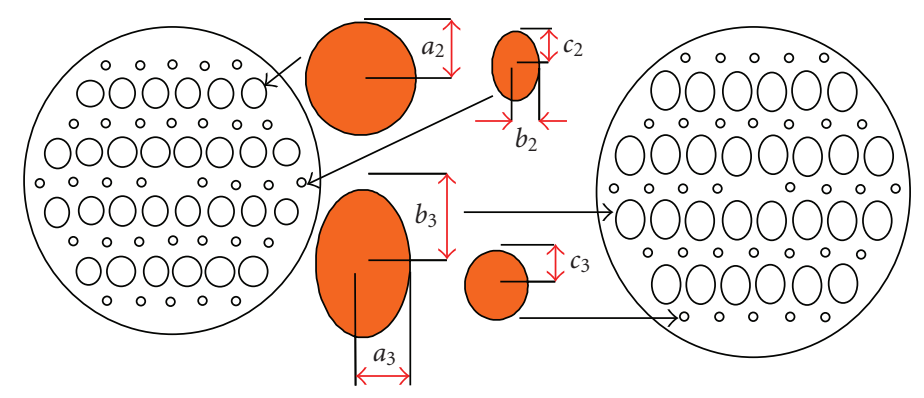

(c)

(d)

FIgURE 1: Cross-section of four types of our proposed PCF structures. (a) Our proposed Type 1. (b) Our proposed Type 2. (c) Our proposed Type 3. (d) Our proposed Type 4.

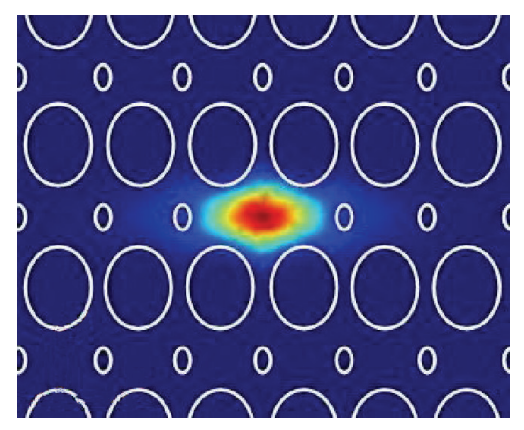

(a) Type 1 structure

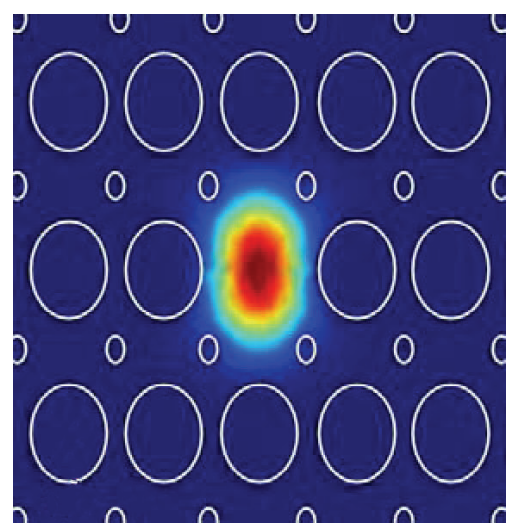

(b) Type 2 structure

Figure 2: Mode field patterns of Type 1 and Type 2.

structure [1]. In addition, the birefringence obtained from Type 2 structure (Figure $1(\mathrm{~b})$ ) is $B=2.5 \times 10^{-3}$, which is lower than those obtained from the structures of Types 1, 3, and 4 .

The effective index and birefringence of PCFs are dependent on the size of air hole and varying wavelengths. As expected, the difference between two polarized direction modes in PCF cladding asymmetry can cause high birefringence in PCFs. Figure 3 shows the birefringence as function of wavelength for the four types of PCFs, whereas the hole spacing remains constant at $\Lambda=1.96 \mu \mathrm{m}, A / \Lambda^{2}=0.4$ (where $A$ is the effective area of air hole in the unit cell), $a_{1} / b_{1}=c_{1} / d_{1}=c_{2} / d_{2}=a_{3} / b_{3}=0.75$, and the ratio $(\eta)$ of large and small air holes fixed with $a_{1} / c_{1}=a_{2} / b_{2}=$ $a_{3} / c_{3}=5.5$. It can be clearly seen in Figure 3 that the birefringence is sensitive to the variation of wavelength $\lambda$, and the birefringences in Types 1, 3, and 4 are much higher than that in Type 2 structure as the value of wavelength is less than $1.5 \mu \mathrm{m}$. When the value of wavelength is larger than $1.6 \mu \mathrm{m}$, the birefringence in Type 3 is much larger than those values from the other three types of structures. The corresponding birefringence of $\lambda=1.55 \mu \mathrm{m}$ for Type 1 is $7.697 \times 10^{-3}$, for Type 2 is $1.502 \times 10^{-3}$, for Type 3 is $8.002 \times 10^{-3}$, and for Type 4 is $6.014 \times 10^{-3}$. In this case, Type 3 structure is superior to the other types on birefringence.

Figure 4 shows the simulation results for the comparison of the birefringences obtained from the four types of structures as a function of effective area of air holes $A / \Lambda^{2}$ in PCF cladding with structure parameters $\Lambda=1.96 \mu \mathrm{m}$, $a_{1} / b_{1}=c_{1} / d_{1}=c_{2} / d_{2}=a_{3} / b_{3}=0.75$; the ratio of large to small air hole size fixed at $\eta\left(a_{1} / c_{1}=a_{2} / b_{2}=a_{3} / c_{3}\right)=5.5$ and the excitation wavelength of $\lambda=1.55 \mu \mathrm{m}$. The results show that the birefringence increases with the effective area $A$. 


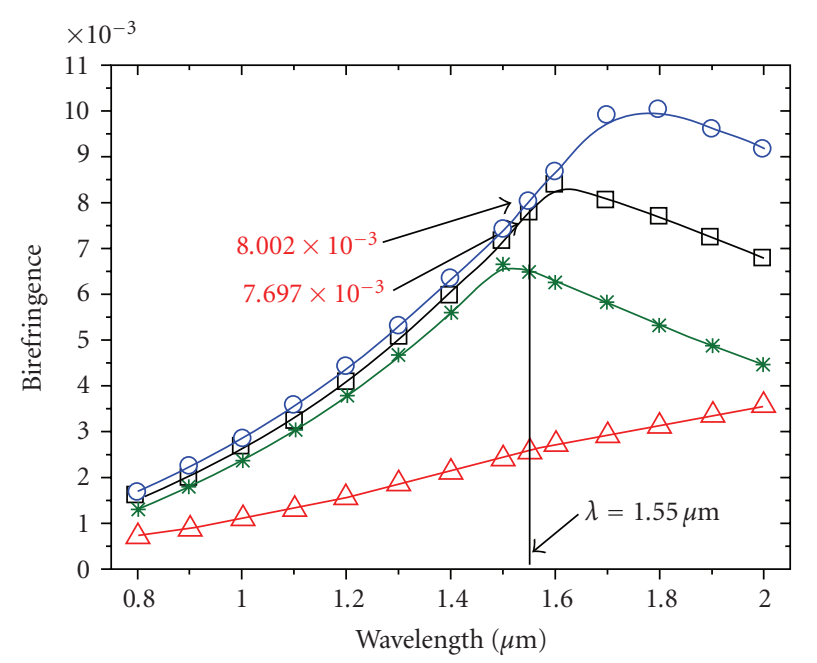

$\begin{array}{ll}\square \text { Our proposed Type } 1 & - \text { Our proposed Type } 3 \\ \triangle \text { Our proposed Type } 2 & * \text { Our proposed Type } 4\end{array}$

FIgURE 3: The birefringence as function of wavelength of four types of PCFs, whereas the hole spacing remains constant at $\Lambda=1.96 \mu \mathrm{m}$, $A / \Lambda^{2}=0.4, a_{1} / b_{1}=c_{1} / d_{1}=c_{2} / d_{2}=a_{3} / b_{3}=0.75$, and the ratio of large and small air holes fixed $\eta\left(a_{1} / c_{1}=a_{2} / b_{2}=a_{3} / c_{3}\right)=5.5$.

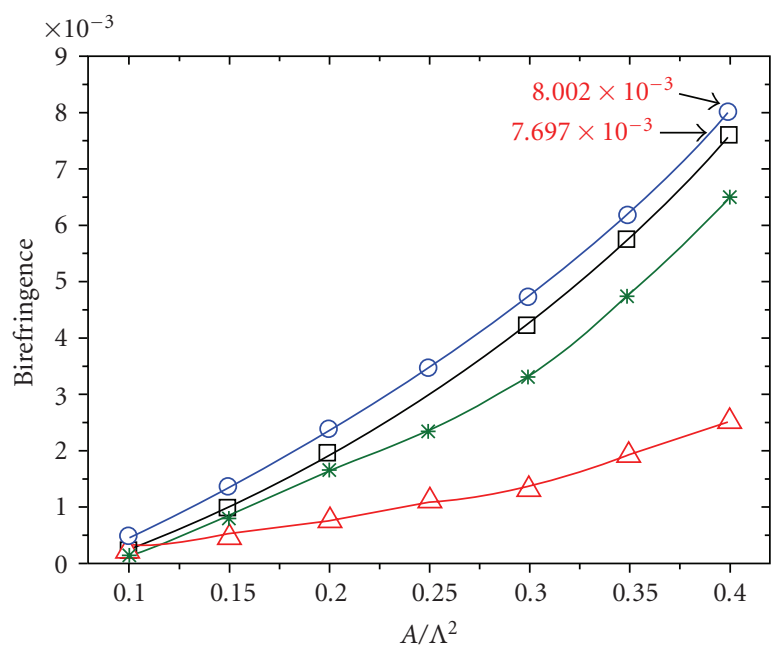

$\begin{array}{ll}\square \text { Our proposed Type } 1 & - \text { Our proposed Type } 3 \\ \triangle \text { Our proposed Type } 2 & \rightarrow \text { Our proposed Type } 4\end{array}$

FIGURE 4: Dependence of modal birefringence on effective area $A / \Lambda^{2}$.

With the increasing effective area $A$ in PCF cladding, the difference of air filling fraction becomes large between $X$ - and $Y$-directions; thus, more confined fields exist in the $Y$-direction. The birefringences of Types 1, 3, and 4 increase quickly as the effective area $A / \Lambda^{2}$ increases. A maximum value of birefringence in Type 3 reaches $8.002 \times 10^{-3}$ at $A / \Lambda^{2}=0.4$. In this case, we can also see that the birefringence of Type 2 is lower than those of the other three types.

The asymmetry as well as the stability on birefringence due to the interruption of the lattice is evident. The field confinement and its decay rate play a fundamental role in the

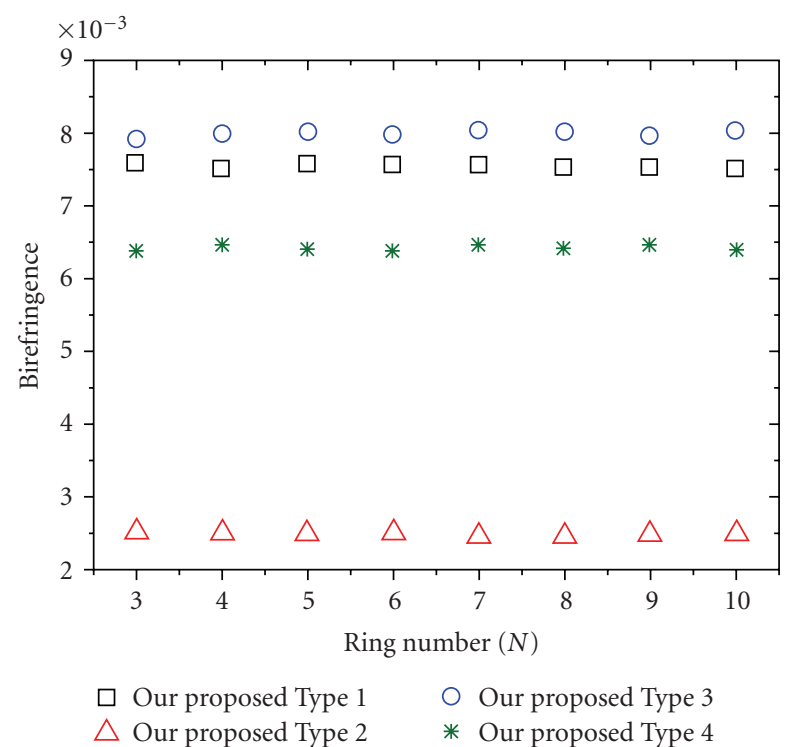

FIgURE 5: Dependence of modal birefringence on number of airhole rings.

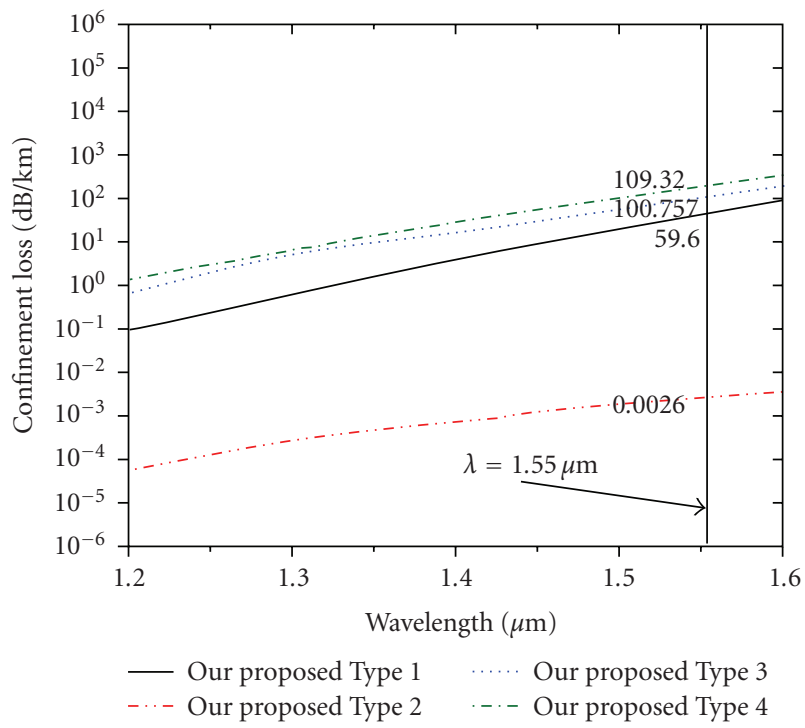

Figure 6: Confinement loss as function of number of rings $(N=$ 4-7).

leakage properties. They depend on the air hole diameter, on their pitch, and on the number of rings. In practice, more than six air holes are often needed to reduce the confinement loss to an acceptable level. For the sake of accuracy, we use 13 rings in this paper. In the following analysis, we fix the wavelength, $\Lambda=1.96 \mu \mathrm{m}, a_{1} / b_{1}=c_{1} / d_{1}=c_{2} / d_{2}=$ $a_{3} / b_{3}=0.75$, the ratio of large and small air holes $\eta\left(a_{1} / c_{1}=\right.$ $\left.a_{2} / b_{2}=a_{3} / c_{3}\right)=5.5$, and the excitation wavelength of $\lambda=1.55 \mu \mathrm{m}$. The influence of asymmetry cladding on the birefringence stability is also significant, which is illustrated in Figure 5. The reason why the birefringences of Types 1 and 3 PCFs is better than that of Types 2 and 4 PCFs as shown in Figure 5 is that the birefringences of Type 1 and 
Type 3 are caused by smaller air holes in the $X$-direction, which give arise to greater fields confined in the core region, thus the birefringence is more stable even in the case of 13 rings.

The FEM is used to calculate the confinement loss of the fundamental modes in the four types of PCFs, and results are plotted in Figure 6. As an example of our interest, the parameters are $A / \Lambda^{2}=0.4, \quad \eta\left(a_{1} / c_{1}=a_{2} / b_{2}=\right.$ $\left.a_{3} / c_{3}\right)=5.5$, and $\lambda=1550 \mathrm{~nm}$. As seen in Figure 6 , with the number of air hole rings increasing, the confinement loss rapidly decreases, meaning that the confinement of the guided mode is significantly improved. Types 3 and 4 PCFs have greater confinement loss, whereas that loss for Type 1 PCF is reduced, and further reduction can be achieved by Type 2 . The confinement losses of Type 2 PCF is $0.026 \mathrm{~dB} / \mathrm{km}$, which is much lower than the values of 109.32 , 100.757, and $59.6 \mathrm{~dB} / \mathrm{km}$ for Types 1, 3, and 4 structures, when the number of the air hole rings is $N=7$. The reason why the confinement loss of Type 2 PCF is much lower than that of Types 1, 3, and 4 PCFs is that the core in Type 2 PCF is better enclosed by the first ring of smaller air holes under the condition of identical effective air-hole area.

\section{CONCLUSIONS}

In conclusion, a high birefringent index-guiding photonic crystal fiber with asymmetric cladding is successfully demonstrated. Four types of our proposed PCFs are investigated. Results show that the birefringences of our proposed Type 1 and Type 3 structures are much higher than those obtained from Type 2 and Type 4 . The reason why the birefringences of Types 1 and 3 PCFs is better than those of Types 2 and 4 PCFs is that the birefringences of Type 1 and Type 3 contribute by smaller air holes in the $X$-direction, which gives arise to more fields confined in the core region. The confinement loss of Type 2 PCF is much lower than those of Types 1, 3 , and 4 structures. This is due to the smaller air holes of the first ring under the condition of identical effective airhole area. The birefringence is achieved by introducing the different air-hole sizes in the triangular structure. We utilize the intrinsically large index contrast in PCFs in combination with asymmetric cladding designs. There are many methods to induce birefringence in photonic crystal fibers. The key point is to destroy the symmetry of the structure, and to create the effective index difference between the two orthogonal polarization states. The suggested structure possesses a large birefringence to separate the two polarization modes. Our simulation results provide valuable insight into realization of PCF with even appreciable birefringence than those demonstrated previously in literature.

\section{ACKNOWLEDGMENTS}

The authors are thankful for the financial support from Ministry of Economic Affairs, Taiwan, under Grant no. NSC 97-2120-M-002-013- and the National Science Council, Taiwan, under Grant no. NSC 96-2112-M-231-001-MY3.

\section{REFERENCES}

[1] A. Ortigosa-Blanch, J. C. Knight, W. J. Wadsworth, et al., "Highly birefringent photonic crystal fibers," Optics Letters, vol. 25, no. 18, pp. 1325-1327, 2000.

[2] Y. Jung, S. R. Han, S. Kim, U. C. Paek, and K. Oh, "Versatile control of geometric birefringence in elliptical hollow optical fiber," Optics Letters, vol. 31, no. 18, pp. 2681-2683, 2006.

[3] P. R. McIsaac, "Symmetry-induced modal characteristics of uniform waveguides-I: summary of results," IEEE Transactions on Microwave Theory and Techniques, vol. 23, no. 5, pp. 421-429, 1975.

[4] T. A. Birks, J. C. Knight, and P. St. J. Russell, "Endlessly singlemode photonic crystal fiber," Optics Letters, vol. 22, no. 13, pp. 961-963, 1997.

[5] C. J. S. de Matos and J. R. Taylor, "Multi-kilowatt, allfiber integrated chirped-pulse amplification system yielding $40 \times$ pulse compression using air-core fiber and conventional erbium-doped fiber amplifier," Optics Express, vol. 12, no. 3, pp. 405-409, 2004.

[6] F. Benabid, J. C. Knight, G. Antonopoulos, and P. St. J. Russell, "Stimulated Raman scattering in hydrogen-filled hollow-core photonic crystal fiber," Science, vol. 298, no. 5592, pp. 399402, 2002.

[7] J. Noda, K. Okamoto, and Y. Sasaki, "Polarization-maintaining fibers and their applications," Journal of Lightwave Technology, vol. 4, no. 8, pp. 1071-1089, 1986.

[8] M. J. Steel and R. M. Osgood Jr., "Polarization and dispersive properties of elliptical-hole photonic crystal fibers," Journal of Lightwave Technology, vol. 19, no. 4, pp. 495-503, 2001.

[9] K. Saitoh and M. Koshiba, "Photonic bandgap fibers with high birefringence," IEEE Photonics Technology Letters, vol. 14, no. 9, pp. 1291-1293, 2002.

[10] A. Hochman and Y. Leviatan, "Modal dynamics in hollowcore photonic-crystal fibers with elliptical veins," Optics Express, vol. 13, no. 16, pp. 6193-6201, 2005.

[11] Y.-F. Chau, H.-H. Yeh, and D. P. Tsai, "Significantly enhanced birefringence of photonic crystal fiber using rotational binary unit cell in fiber cladding," Japanese Journal of Applied Physics, vol. 46, no. 41-44, pp. L1048-L1051, 2007.

[12] Y.-S. Sun, Y.-F. Chau, H.-H. Yeh, L.-F. Shen, T.-J. Yang, and D. P. Tsai, "High birefringence photonic crystal fiber with a complex unit cell of asymmetric elliptical air hole cladding," Applied Optics, vol. 46, no. 22, pp. 5276-5281, 2007.

[13] Y.-S. Sun, Y.-F. Chau, H.-H. Yeh, and D. P. Tsai, "Highly birefringent index-guiding photonic crystal fiber with squeezed differently sized air-holes in cladding," Japanese Journal of Applied Physics, vol. 47, no. 5, part 1, pp. 3755-3759, 2008.

[14] M. J. Steel and R. M. Osgood Jr., "Elliptical-hole photonic crystal fibers," Optics Letters, vol. 26, no. 4, pp. 229-231, 2001.

[15] D. Chen and L. Shen, "Highly birefringent elliptical-hole photonic crystal fibers with double defect," Journal of Lightwave Technology, vol. 25, no. 9, pp. 2700-2705, 2007.

[16] D. Chen and L. Shen, "Ultrahigh birefringent photonic crystal fiber with ultralow confinement loss," IEEE Photonics Technology Letters, vol. 19, no. 4, pp. 185-187, 2007.

[17] T. P. Hansen, J. Broeng, S. E. B. Libori, et al., "Highly birefringent index-guiding photonic crystal fibers," IEEE Photonics Technology Letters, vol. 13, no. 6, pp. 588-590, 2001.

[18] A. Ortigosa-Blanch, J. C. Knight, W. J. Wadsworth, et al., "Highly birefringent photonic crystal fibers," Optics Letters, vol. 25, no. 18, pp. 1325-1327, 2000. 
[19] T. P. Hansen, J. Broeng, S. E. B. Libori, et al., "Highly birefringent index-guiding photonic crystal fibers," IEEE Photonics Technology Letters, vol. 13, no. 6, pp. 588-590, 2001.

[20] P. R. Chaudhuri, V. Paulose, C. Zhao, and C. Lu, "Nearelliptic core polarization-maintaining photonic crystal fiber: modeling birefringence characteristics and realization," IEEE Photonics Technology Letters, vol. 16, no. 5, pp. 1301-1303, 2004.

[21] J. C. Knight, T. A. Birks, P. St. J. Russell, and D. M. Atkin, "All-silica single-mode optical fiber with photonic crystal cladding," Optics Letters, vol. 21, no. 19, pp. 1547-1549, 1996.

[22] V. Mocella, "Negative refraction in photonic crystals: thickness dependence and Pendellösung phenomenon," Optics Express, vol. 13, no. 5, pp. 1361-1367, 2005.

[23] A. Ferrando, E. Silvestre, J. J. Miret, P. Andrés, and M. V. Andrés, "Full-vector analysis of a realistic photonic crystal fiber," Optics Letters, vol. 24, no. 5, pp. 276-278, 1999.

[24] D. Mogilevtsev, T. A. Birks, and P. St. J. Russell, "Localized function method for modeling defect modes in 2-D photonic crystals," Journal of Lightwave Technology, vol. 17, no. 11, pp. 2078-2081, 1999.

[25] Z. Zhu and T. G. Brown, "Multipole analysis of hole-assisted optical fibers," Optics Communications, vol. 206, no. 4-6, pp. 333-339, 2002.

[26] Z. Zhu and T. G. Brown, "Full-vectorial finite-difference analysis of microstructured optical fibers," Optics Express, vol. 10, no. 17, pp. 853-864, 2002.

[27] F. Brechet, J. Marcou, D. Pagnoux, and P. Roy, "Complete analysis of the characteristics of propagation into photonic crystal fibers, by the finite element method," Optical Fiber Technology, vol. 6, no. 2, pp. 181-191, 2000.

[28] Z. Zhu and T. G. Brown, "Stress-induced birefringence in microstructured optical fibers," Optics Letters, vol. 28, no. 23, pp. 2306-2308, 2003.

[29] A. Cucinotta, S. Selleri, L. Vincetti, and M. Zoboli, "Holey fiber analysis through the finite-element method," IEEE Photonics Technology Letters, vol. 14, no. 11, pp. 1530-1532, 2002. 

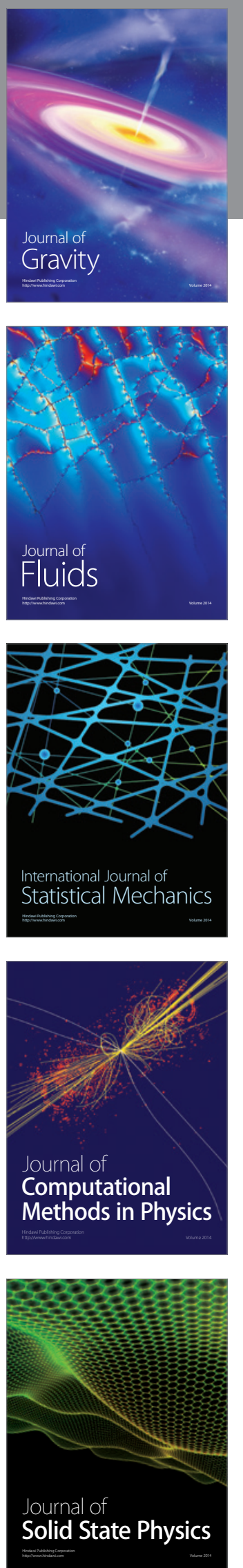

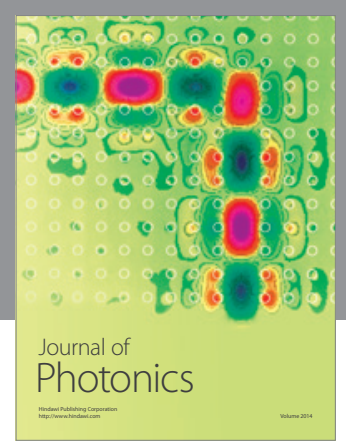

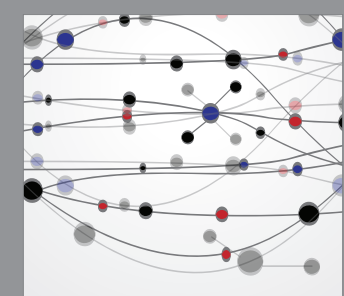

The Scientific World Journal
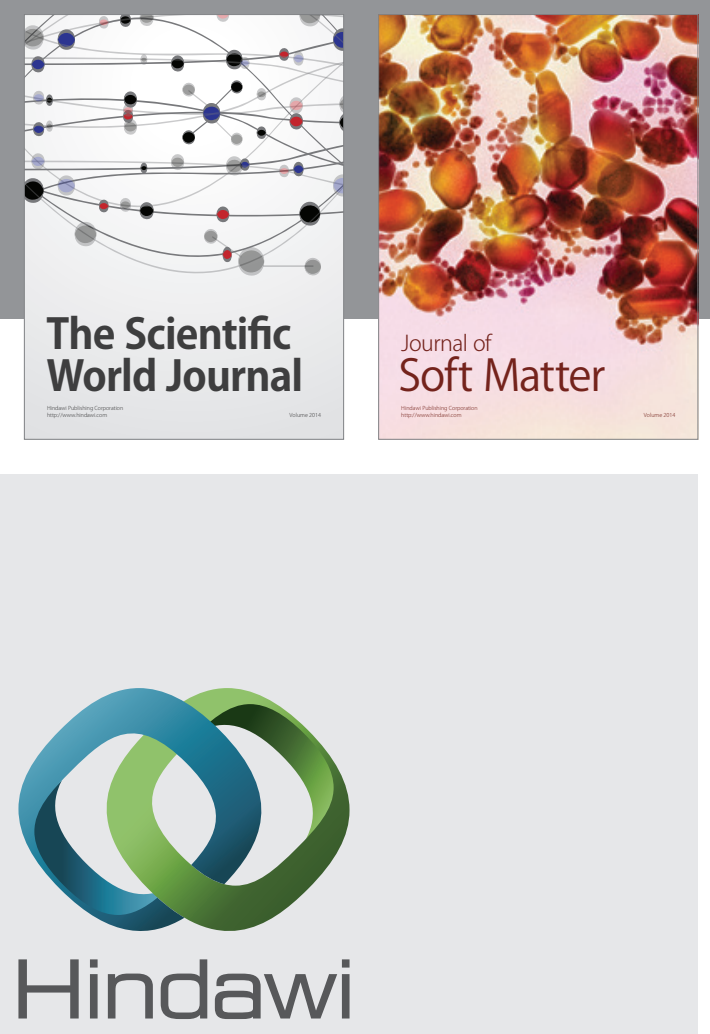

Submit your manuscripts at

http://www.hindawi.com
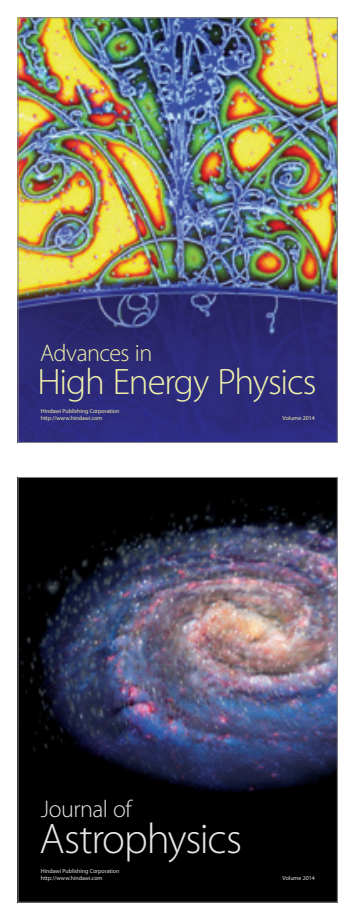
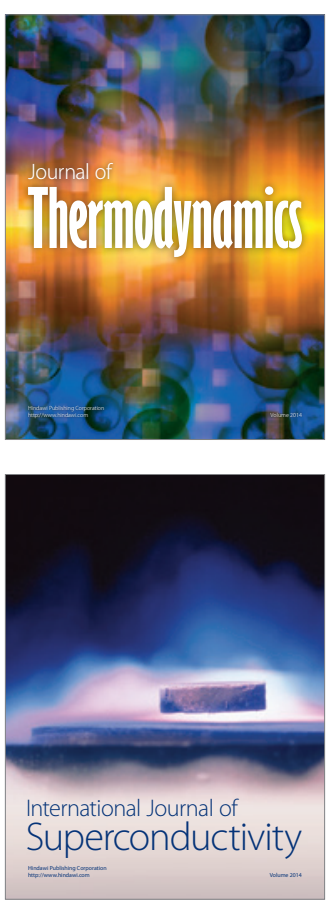
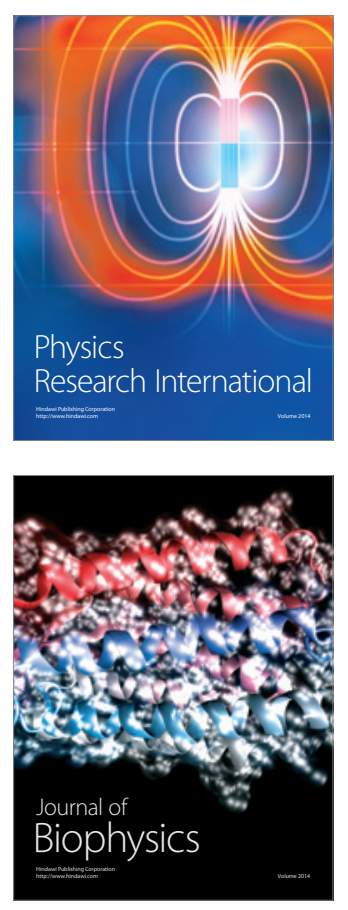
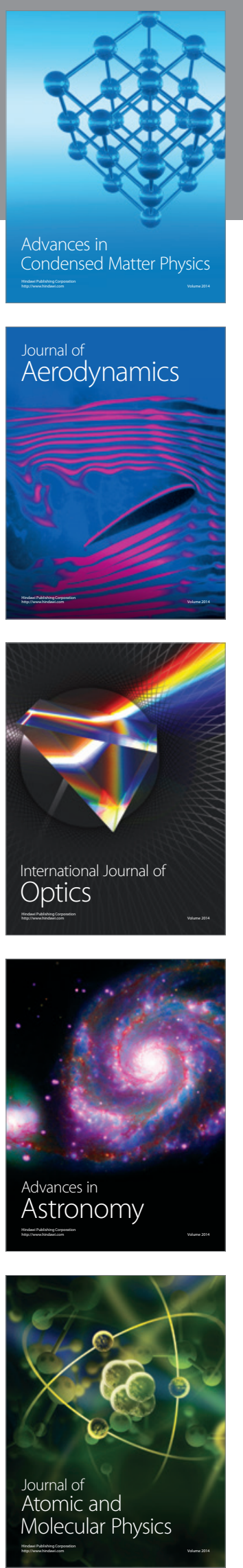